\title{
Reticular patterned episcleral venous plexus and 360-degree episcleral venous fluid wave after hemi- gonioscopy assisted transluminal trabeculotomy
}

\author{
Julia Wiens ${ }^{1}$, Malcolm Gooi ${ }^{2}$, Matt Schlenker ${ }^{3}$, Teong Lam Gooi², Danielle Wentzell², Patrick Gooi' \\ 'Max Rady College of Medicine, University of Manitoba, Winnipeg R3E OW2, Canada. \\ ${ }^{2}$ Cloudbreak Eye Care, Calgary T2H OC8, Canada. \\ ${ }^{3}$ Department of Ophthalmology and Vision Sciences, University of Toronto, Toronto M5T 3A9, Canada. \\ ${ }^{4}$ Division of Ophthalmology, Department of Surgery, University of Calgary, Calgary T2V 1P9, Canada. \\ Correspondence to: Dr. Patrick Gooi, Cloudbreak Eye Care, Suite 315-5340 1st ST SW, Calagry, Alberta T2HOC8, Canada. \\ E-mail:Patrick.gooi@cloudbreak.ca

\begin{abstract}
How to cite this article: Wiens J, Gooi M, Schlenker M, Gooi TL, Wentzell D, Gooi P. Reticular patterned episcleral venous plexus and 360-degree episcleral venous fluid wave after hemi-gonioscopy assisted transluminal trabeculotomy. Mini-invasive Surg 2021;5:8. http://dx.doi.org/10.20517/2574-1225.2020.105
\end{abstract}

Received: 15 Nov 2020 First Decision: 7 Dec 2020 Revised: 31 Dec 2020 Accepted: 8 Jan 2021 Published: 3 Feb 2021

Academic Editor: Kazuyuki Hirooka Copy Editor: Xi-Jun Chen Production Editor: Yue-Yue Zhang

\begin{abstract}
We describe a method for eliciting an episcleral venous fluid wave (EVFW) in eyes presenting with reticular patterned episcleral venous plexus, after a hemi-gonioscopy assisted transluminal trabeculotomy (hemi-GATT). To reduce the risk of post-operative hyphema and reduce intraoperative tissue manipulation, a hemi-GATT (targeting 180-degrees of Schlemm's canal) was performed. Post-hemi-GATT, the ability to inject balanced salt solution and obtain an EVFW in both the treated (inferior) and untreated (superior) sectors of the eye supports the surgical success of the technique, and demonstrates an enhanced fluid outflow and subsequent vessel blanching. The pre-operative intraocular pressure of $20 / 21 \mathrm{mmHg}$ in a single subject decreased to $18-, 12-$ and $15-\mathrm{mmHg}$ after one day, one month and 3 months post-op, respectively, and the subject was rendered medication-free. This method of performing a hemi-GATT to effectively obtain an EVFW provides evidence for novel treatment algorithms in patients with a reticular episcleral venous plexus where identification of major outflow vessels is less apparent.
\end{abstract}

Keywords: Gonioscopy assisted transluminal trabeculotomy, micro-invasive glaucoma surgeries, glaucoma surgery, episcleral venous fluid wave 


\section{INTRODUCTION}

Glaucoma stands as the leading cause of irreversible blindness worldwide and approximately $3 \%$ of the population between 40-80 years old have a primary open angle glaucoma ${ }^{[1]}$. While a trabeculectomy is considered as the gold standard treatment for glaucoma management, the use of micro-invasive glaucoma surgeries (MIGS) for the treatment of mild to moderate glaucoma are growing due to their high safety profile, rapid recovery time, and minimally invasive nature ${ }^{[2,3]}$. Canal-based MIGS procedures attempt to bypass the trabecular meshwork, a major site of resistance to aqueous humor drainage, and enhance the conventional outflow ${ }^{[4]}$. While the true efficacy of MIGS procedures is still to be elucidated, surgeons are discovering that device design and surgical expertise are not the sole determinants of treatment success. The placement of these devices in terms of optimal orientation to best enhance aqueous outflow is of paramount importance, giving rise to the term "targeted MIGS". Implantation of iStents (Glaukos Corporation, Laguna Hills, CA, USA) is one such canal-based procedure. However, current literature supports implantation of one to three iStents in areas of dense trabecular pigmentation, adjacent to major aqueous and episcleral veins that are identified via external examination and/or in areas of focal blood reflux in the Schlemm canal as seen with gonioscopy, in an attempt to target the major collector channel ostia in anterior segments ${ }^{[4]}$. The rationale is to target these large capacity veins to effectively enhance aqueous drainage. We describe a reticular patterned episcleral venous plexus that comprises of a network of numerous small-caliber, finer vessels, rather than a few, large-caliber vessels.

Gonioscopy assisted transluminal trabeculotomy (GATT) is a novel, ab interno MIGS approach to a 360-degree trabeculotomy that is conjunctival-sparing while also resulting in successful reductions in intraocular pressure (IOP) and decreased need for glaucoma medications ${ }^{[5]}$. A hemi-GATT unroofs 180-degrees of the Schlemm's canal to reduce the risk of postoperative hyphema and reduces intraoperative tissue manipulation. Both a 360 degree-GATT and hemi-GATT aim to improve aqueous outflow through Schlemm's canal and adjacent collector channels ${ }^{[5]}$. Unlike in filtration surgery where bleb morphology correlates with surgical success, to date there is no concrete evidence, whether pre-operative, intraoperative or post-operative, of a similar association between bleb morphology and canal-based MIGS surgery ${ }^{[3]}$. However, growing evidence supports the correlation between the presence of an episcleral venous fluid wave (EVFW) and post-operative reductions in IOP as well as the need for fewer glaucoma medications and/or additional surgeries ${ }^{[3]}$. An EVFW is an intraoperative technique performed at the conclusion of a surgery wherein diffuse vessel blanching is achieved by injecting balanced salt solution (BSS) that flows into the conjunctival and episcleral venous systems, demonstrating possible patency of the conventional aqueous outflow system ${ }^{[3,6]}$. For an EVFW to be present, fluid must be able to travel from collector channel openings, through the deep and mid scleral plexuses to the episcleral plexus terminating in the conjunctival veins, thereby demonstrating an enhanced aqueous outflow ${ }^{[3]}$.

Due to the broad 180- to 360-degree area of treatment with hemi-GATT and GATT procedures respectively, theoretically, one could propose that these procedures could be effective even in eyes demonstrating a reticular patterned episcleral venous plexus as one could target large areas of Schlemm's canal rather than individual veins to enhance aqueous drainage. We propose that hemi-GATT is an effective technique to enhance aqueous outflow in eyes that have a reticular patterned episcleral venous plexus, with EVFW serving as an indicator of probable surgical success.

\section{CASE REPORT}

\section{Description of the surgical technique}

Using standard sterile eye preparation, the surgical eye was draped and held open with a wire lid speculum. The inferior sector of the eye (inferior 180-degrees) was selected as the hemi-GATT target. The hemi-GATT was performed using the ripcord technique, modified from that described by Grover et al. ${ }^{[5]}$ [Figure 1, Video 1]. 


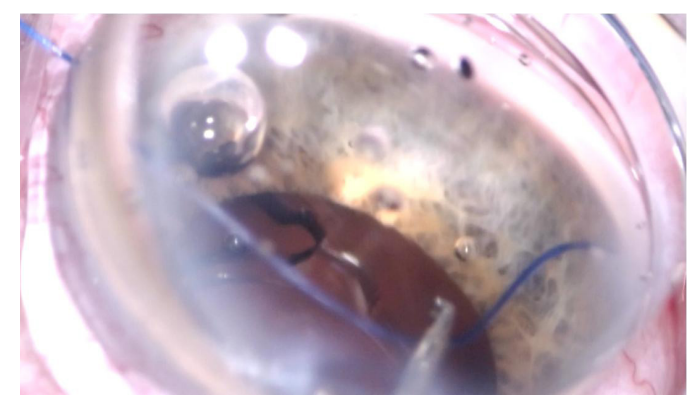

Figure 1. Hemi-gonioscopy assisted transluminal trabeculotomy performed with ripcord technique in the inferior sector.

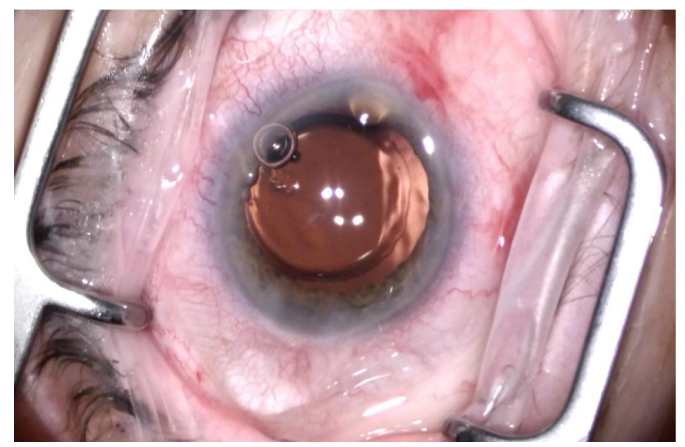

Figure 2. External ocular examination post-hemi-gonioscopy assisted transluminal trabeculotomy showed a reticular episcleral venous pattern in the superior and inferior sectors. Note that the reticular pattern is more pronounced in the superior sector (left of image).

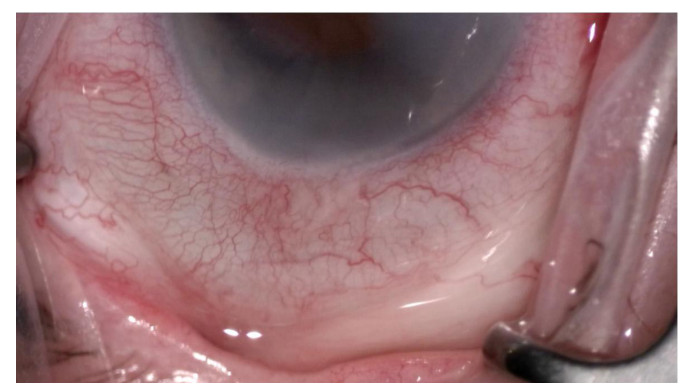

Figure 3. Reducing the intraocular pressure results in engorgement of the episcleral venous plexus superiorly and inferiorly.

The procedure for EVFW generation, as described below, was based on that described by Fellman et al. ${ }^{[3,6]}$.

Post-hemi-GATT, the gonioscopy lens was removed for external examination of the episcleral vasculature both adjacent to the treated site (inferior sector) and in the untreated superior sector. The reticular pattern of the episcleral venous plexus was noted in both sectors [Figure 2]. In the mid-anterior chamber, an irrigation/aspiration probe was used to remove the residual viscoelastic substance that was left behind by the hemi-GATT at an irrigation pressure of $65 \mathrm{mmHg}$. When closely observing the adjacent episcleral veins, the IOP in the anterior chamber was reduced by halting fluid irrigation until episcleral veins filled with blood and focal blood reflux was seen into the anterior chamber adjacent to the surgical site (inferior sector). At this point, there was maximal prominence of the reticular episcleral venous pattern in both the superior and inferior sectors [Figure 3]. Toward the end of the surgery, at the time of BSS injection, the episcleral vessels were closely observed for vessel blanching from the BSS washout. Hyperinfusion with BSS post-hemi-GATT created an EVFW with progressive vessel blanching in both the treated inferior 


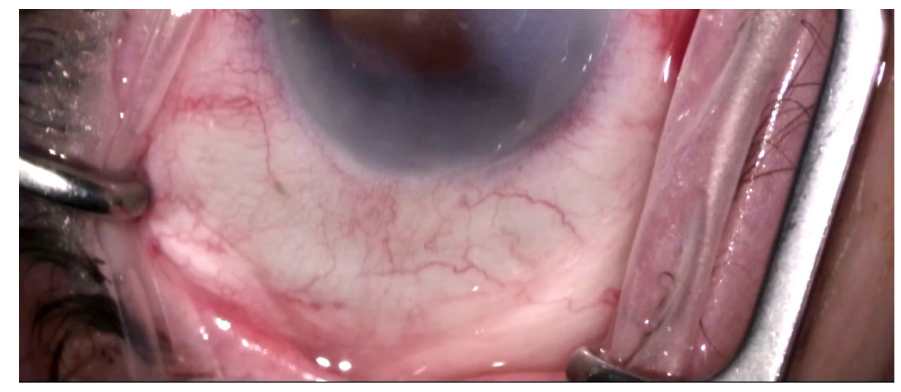

Figure 4. Hyperinfusion of the anterior chamber with balanced salt solution causes an episcleral venous fluid wave and blanching of both the superior and inferior sectors.

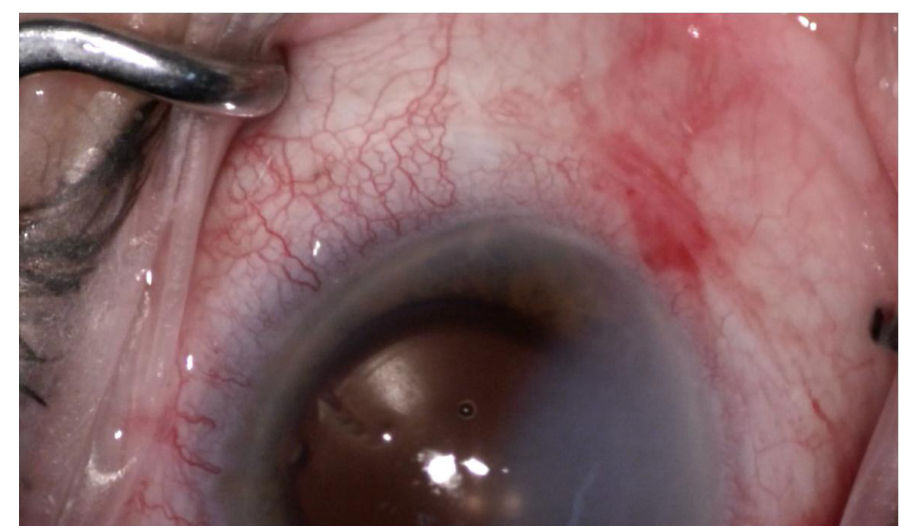

Figure 5. Reducing the intraocular pressure to near-physiologic conditions results in a prominent reticular episcleral venous pattern in the superior, untreated sector (left of image). Note that there is still some residual blanching in the inferior, treated sector (right of image).

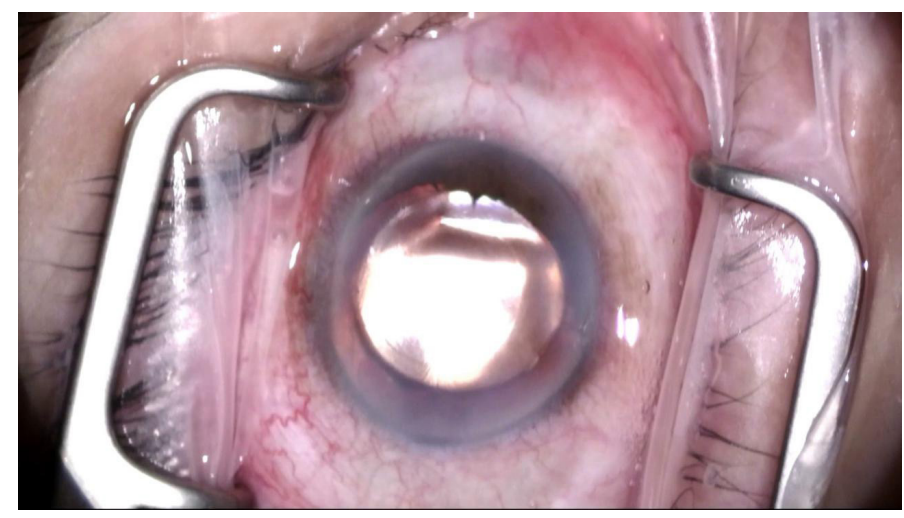

Figure 6. Hyperinflation of the anterior chamber with balanced salt solution to supraphysiologic conditions results in a 360-degree limbal blanching during the episcleral venous fluid wave.

and untreated superior sectors [Figure 4]. Reducing IOP to near-physiologic conditions resulted in a prominent reticular episcleral venous pattern in the superior, untreated sector; residual blanching remained in the treated, inferior sector [Figure 5]. Repeated hyperinflation of the anterior chamber with BSS to supraphysiologic conditions results in 360-degree limbal blanching during EVFW [Figure 6], and when IOP is lowered, there was a 360-degree engorgement of the episcleral venous plexus [Figure 7].

\section{Results/Case study}

This procedure was performed on the right eye of a 58-year-old female with pigmentary glaucoma in both eyes. The pre-operative IOP was $20 / 21 \mathrm{mmHg}$ as measured with Goldmann tonometry, and the subject 


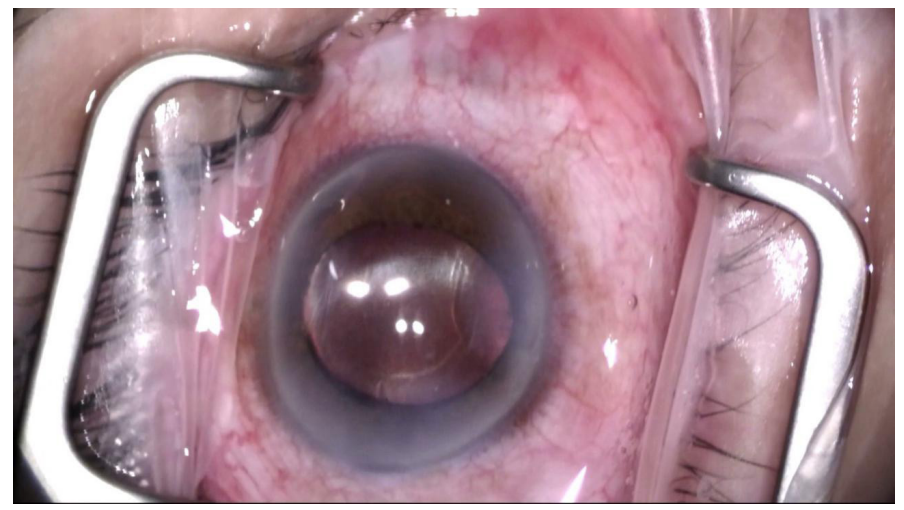

Figure 7. When the intraocular pressure is lowered, there is a 360-degree engorgement of the episcleral venous plexus.

took topical pressure-lowering medication of travaprost, once daily. Prior to the surgery, external ocular examination revealed a reticular episcleral venous plexus with a diffuse interconnected meshwork of veins and venules. The post-operative IOP decreased to $18 \mathrm{mmHg}, 12 \mathrm{mmHg}$ and $15 \mathrm{mmHg}$, on day one, one month and 3 months post-op, respectively. At 3 months post-op, the patient was not taking any pressurelowering medications. Blanching of the episcleral venous plexus due to the EVFW was observed postoperatively in both the superior and inferior sectors.

\section{DISCUSSION}

Glaucoma is the leading cause of irreversible blindness worldwide with an increasing prevalence in the aging population $^{[1]}$. When a surgical treatment is desired to halt or slow down the disease progression, canal-based MIGS surgeries are typically favored ${ }^{[6]}$. The focus of these procedures is to enhance the physiological aqueous outflow, generally as an alternative to those requiring formation of an artificial external bleb ${ }^{[6]}$. Canal-based MIGS procedures attempt to bypass the area of greatest resistance to aqueous outflow, the trabecular meshwork, and thus enhance aqueous drainage ${ }^{[1,7]}$. MIGS devices like the iStent (Glaukos Corporation, Laguna Hills, CA, USA) are heparin-coated titanium stents, designed to enhance aqueous outflow through the conventional outflow pathway ${ }^{[2]}$. However, to be effective, the stent must be placed to target large capacity veins that support drainage directly from Schlemm's canal rather than from smaller venules that drain distal plexuses ${ }^{[2,8]}$. Recent studies suggest that implantation of two iStents, instead of three, results in similar reductions in IOP, inferring therefore that proper device placement rather than the device number most likely dictates surgical success ${ }^{[1]}$. However, a problem arises when patients present with a reticular patterned episcleral venous plexus because identifying the ideal target for iStent implantation becomes increasingly difficult.

Reticular episcleral venous patterning is seen as an interconnected meshwork of veins and venules where clear large capacity outflow veins cannot be easily identified. This is in contrast to detecting discrete, largecaliber episcleral veins. To the best of our knowledge, other in vivo patterns of the episcleral venous plexus have not yet been defined. In eyes with a reticular patterned episcleral venous plexus, it would be possible to implant numerous iStents, approximately 1-2 clock hours apart, spanning the venous plexus. If the surgeon uses a direct view gonioprism that requires tilting of the patient's head and microscope, implanting more than 3 iStents can become surgically challenging because the easiest access from a temporal approach is the nasal 180 degrees of Schlemm's canal. Furthermore, the cost of the surgery increases with each additional implanted device. Potentially, using iStent inject devices combined with a direct-view gonio mirror that does not require tilting the microscope allows one to treat the full 360-degrees of the trabecular meshwork. As demonstrated in this case study [Figures 6 and 7], we achieved a full 360-degree EVFW with a 180-degree unroofing of Schlemm's canal with a hemi-GATT. 
As shown, eyes presenting with a reticular patterned episcleral venous plexus are good candidates for procedures like the hemi-GATT that target a larger sector of Schlemm's canal. Not only was there a reduction in IOP, but there was also an elimination of medication burden associated with glaucoma. Furthermore, since the subject presented with a diffuse reticular pattern, one might have been tempted to perform a GATT to target the entire drainage system circumference. However, previous preliminary results comparing the success rates for the GATT and hemi-GATT show no significant difference (success rate of $74 \%$ for GATT and $70 \%$ hemi-GATT) ${ }^{[9]}$. Herein, the hemi-GATT was shown to enhance drainage in both the superior and inferior sectors while preserving the superior 180-degrees of Schlemm's canal to enable future angular surgery, if needed.

When determining the approach to performing a hemi-GATT, a surgeon must choose the sector to target. Due to the ease of surgical access, the nasal quadrant is most commonly favored as the location for Schlemm's canal surgeries. However, surgical ease is not the sole reason for this target. The nasal quadrant is also the location of the highest density of collector channels ${ }^{[2]}$. With 25-35 collector channels per eye, one desires to target as many of these as possible when performing a hemi-GATT to maximize conventional aqueous flow ${ }^{[3]}$. To exit the eye via the conventional pathway, aqueous fluid must pass through the collector channels for subsequent drainage into the deep venous scleral plexus, the mid scleral plexus and the episcleral plexus to finally reach the conjunctival veins ${ }^{[3]}$. While no clinically established marker has yet been proven to conclusively predict the likelihood of success with canal-based MIGS procedures, growing evidence supports the presence of an EVFW as a marker of surgical success ${ }^{[3,6]}$. Research has shown that the ability to elicit a pronounced EVFW with diffuse blanching of the visible vessels correlates with the need for fewer medications and lower postoperative $\mathrm{IOP}^{[3]}$. It is theorized that eyes with a positive EVFW must have patent collector channels and a downstream outflow system for the infused BSS to blanch the episcleral vessels ${ }^{[3]}$. As demonstrated here, the inferior 180 -degrees incorporating the infra-nasal quadrant serves as an optimal location for hemi-GATT to best enhance aqueous outflow. Despite leaving the superior 180-degrees of the eye untreated, diffuse episcleral venous blanching in this area was observed with BSS infusion. In some patients, treatment of a section of Schlemm's canal may be sufficient to achieve the desired surgical outcome.

In conclusion, a hemi-GATT targeting the inferior 180-degrees of Schlemm's canal is a MIGS procedure that is applicable to eyes demonstrating a reticular pattern episcleral venous plexus. The ability to elicit a pronounced EVFW post-hemi-GATT that was seen in both the superior untreated sector and the inferior treated sector, indicates patency of the collector channels and enhanced aqueous outflow via the conventional outflow pathway. Further work may help determine the ideal glaucoma surgical procedure based on a patient's particular episcleral venous pattern.

\section{DECLARATIONS}

\section{Authors' contributions}

Made substantial contributions to conception and design of the study, and performed data analysis and interpretation: Wiens J, Gooi M, Schlenker M, Gooi TL, Wentzell D, Gooi P

\section{Availability of data and materials}

Not applicable.

\section{Financial support and sponsorship}

None. 


\section{Conflicts of interest}

Patrick Gooi is a consultant for Alcon, Allergan, Bausch and Lomb, Glaukos, and Santen. He has a patent on a glaucoma device.

Matt Schlenker is a consultant for Abbvie, Alcon, Bausch Health, J\&J, Thea-Labtician, Santen, and Zeiss.

\section{Ethical approval and consent to participate}

Research was performed in accordance with the Declaration of Helsinki and approved by the Health Research Ethics Board of Alberta.

\section{Consent for publication}

Not applicable.

\section{Copyright}

(c) The Author(s) 2021.

\section{REFERENCES}

1. Agrawal P, Bradshaw SE. Systematic literature review of clinical and economic outcomes of micro-invasive glaucoma surgery (MIGS) in primary open-angle glaucoma. Ophthalmol Ther 2018;7:49-73.

2. Saheb H, Ahmed II. Micro-invasive glaucoma surgery: current perspectives and future directions. Curr Opin Ophthalmol 2012;23:96104.

3. Fellman RL, Feuer WJ, Grover DS. Episcleral venous fluid wave correlates with trabectome outcomes: intraoperative evaluation of the trabecular outflow pathway. Ophthalmology 2015;122:2385-91.

4. Bostan C, Harasymowycz P. Episcleral venous outflow: a potential outcome marker for iStent surgery. J Glaucoma 2017;26:1114-9.

5. Grover DS, Godfrey DG, Smith O, Feuer WJ, Montes de Oca I, Fellman RL. Gonioscopy-assisted transluminal trabeculotomy, ab interno trabeculotomy: technique report and preliminary results. Ophthalmology 2014;121:855-61.

6. Fellman RL, Grover DS. Episcleral venous fluid wave: intraoperative evidence for patency of the conventional outflow system. $J$ Glaucoma 2014;23:347-50.

7. Richter GM, Coleman AL. Minimally invasive glaucoma surgery: current status and future prospects. Clin Ophthalmol 2016;10:189-206.

8. Grieshaber MC, Pienaar A, Olivier J, Stegmann R. Clinical evaluation of the aqueous outflow system in primary open-angle glaucoma for canaloplasty. Invest Ophthalmol Vis Sci 2010;51:1498-504.

9. Arora A, Nazarali S, Cote S, Duimering A, Schlenker M, Ford B, et al. Gonioscopy assisted transluminal trabeculotomy (GATT): a hemispheric approach. The Canadian Ophthalmologial Society 2018 in Glaucoma Research: the cutting edge symposium, Toronto, Canada, June 2018. Available from: https://cos-sco.ca/toronto2018/wp-content/uploads/2018/04/AbstractBooklet-PaperPresentations.pdf. [Last accessed on 25 Jun 2021] 